\title{
(6) OPEN ACCESS \\ HIV and lower risk of multiple sclerosis: beginning to unravel a mystery using a record-linked database study
}

\author{
Julian Gold, ${ }^{1,2}$ Raph Goldacre, ${ }^{3}$ Hubert Maruszak, ${ }^{1,2}$ Gavin Giovannoni, ${ }^{2}$ \\ David Yeates, ${ }^{3}$ Michael Goldacre ${ }^{3}$
}

- Additional material is published online only. To view please visit the journal online (http://dx.doi.org/10.1136/ jnnp-2014-307932)

${ }^{1}$ The Albion Centre, Prince of Wales Hospital, Sydney, New South Wales, Australia ${ }^{2}$ Centre for Neuroscience and Trauma, The Blizard Institute of Cell and Molecular Science, Queen Mary University of London, London, UK ${ }^{3}$ Unit of Health-Care Epidemiology, Nuffield Department of Population Health, University of Oxford, Oxford, UK

\section{Correspondence to} Dr J Gold, The Albion Centre, Prince of Wales Hospital, Sydney, NSW 2029, Australia; julian.gold@sesiahs.health. nsw.gov.au

Received 20 February 2014 Revised 13 June 2014 Accepted 14 June 2014 Published Online First 4 August 2014

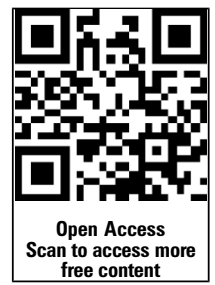

\section{SLinked}

- http://dx.doi.org/10.1136/ jnnp-2014-308297

\section{CrossMark}

To cite: Gold J, Goldacre R, Maruszak $\mathrm{H}$, et al. J Neurol Neurosurg Psychiatry 2015:86:9-12.

\section{ABSTRACT}

Objectives Even though multiple sclerosis (MS) and HIV infection are well-documented conditions in clinical medicine, there is only a single case report of a patient with MS and HIV treated with HIV antiretroviral therapies. In this report, the patient's MS symptoms resolved completely after starting combination antiretroviral therapy and remain subsided for more than 12 years. Authors hypothesised that because the pathogenesis of MS has been linked to human endogenous retroviruses, antiretroviral therapy for HIV may be coincidentally treating or preventing progression of MS. This led researchers from Denmark to conduct an epidemiological study on the incidence of MS in a newly diagnosed HIV population (5018 HIV cases compared with 50149 controls followed for 31875 and 393871 person-years, respectively). The incidence rate ratio for an HIV patient acquiring MS was low at $0.3(95 \% \mathrm{Cl}$ 0.04 to 2.20) but did not reach statistical significance possibly due to the relatively small numbers in both groups. Our study was designed to further investigate the possible association between HIV and MS.

Methods We conducted a comparative cohort study accessing one of the world's largest linked medical data sets with a cohort of 21207 HIV-positive patients and 5298496 controls stratified by age, sex, year of first hospital admission, region of residence and socioeconomic status and 'followed up' by record linkage.

Results Overall, the rate ratio of developing MS in people with HIV, relative to those without HIV , was 0.38 (95\% Cl 0.15 to 0.79$)$.

Conclusions HIV infection is associated with a significantly decreased risk of developing MS. Mechanisms of this observed possibly protective association may include immunosuppression induced by chronic HIV infection and antiretroviral medications.

\section{INTRODUCTION}

Even though multiple sclerosis (MS) and HIV infection are two of the most documented conditions in clinical medicine, with more than one million peerreviewed papers combined, there is only a single case report of a patient with MS and HIV treated with HIV antiretroviral therapies. ${ }^{1}$ In this case report, the patient's MS symptoms resolved completely after starting combination antiretroviral therapy (cART) and remain subsided for more than 12 years of follow-up. In the report, the authors hypothesised that because the pathogenesis of MS has been linked to several human endogenous retroviruses (HERVs), ${ }^{2}{ }^{3}$ antiretroviral therapy for HIV may be coincidentally treating or preventing progression of MS. Publication of this report led investigators from Denmark to conduct an analysis of the incidence of MS in a newly diagnosed HIV population by utilising the Danish National Registry of Patients (1994-2011), which is linked to Civil Registration System and the Danish MS Registry. Their analysis determined that among 5018 first presenting HIV patients and 50149 controls, matched for age and sex and followed for 31875 and 393871 person-years, respectively, the incidence rate ratio (IRR) for an HIV patient acquiring MS was 0.3 (95\% CI 0.04 to 2.20 ). This IRR did not reach statistical significance, possibly due to the relatively small numbers of people who developed MS in each group. ${ }^{4}$ These authors also suggested that HIV therapies may be coincidentally ameliorating the MS and that, if so, this could provide an explanation for the relatively large observed protective effect of HIV on the development of MS. This was the first record linkage study exploring an association between HIV and MS. In order to further test this hypothesis, we explored one of the world's largest linked medical data sets to investigate the possible relationship between HIV and developing MS.

\section{METHODS}

We analysed a national linked data set of English Hospital Episode Statistics (HES) to identify all people with HIV who were discharged from National Health Service hospitals, between 1999 and 2011, as a hospital day-patient or inpatient, irrespective of the reason for this contact. HIV was recorded on the chart, even though the contact may have been for a condition unrelated to HIV. Successive discharge records relating to the same person were linked together as a cumulative statistical record for that person, and these were linked to any death record. HES was provided by the English National Health and Social Care Information Centre; death records were provided by the Office for National Statistics. All data were anonymised by encryption of personal identifiers before being supplied to the Oxford study team for record linkage. The statistical analysis was carried out using analytical software designed within the Oxford research unit using the statistical analysis software package SAS (release V.9.2, SAS Institute 
Inc, Cary, North Carolina, USA). Additional statistical methodology is explained more fully and referenced in the online supplementary appendix.

The earliest record of HIV in HES was the record used for analysis. Having identified all people in the data set with HIV, we stratified them into 5-year age bands, sex, year of first hospital admission, region of residence and quintiles of Index of Multiple Deprivation (a standard national measure of socioeconomic status). We then 'followed up' these people, through record linkage until 31 December 2011, for any subsequent record of MS. Anyone who had a record of MS prior to or concurrent with the HIV record was excluded from the cohort. This excluded five people with prior MS and three people with a simultaneous record of HIV and MS.

For comparison, we also identified a cohort of people without HIV. These were hospital controls selected from our data set; they comprised all people in HES from 1999 to 2011 whose principal reason for an episode of care was for a minor medical or surgical condition or injury (the earliest, for any individual person, of any of those listed in the footnote to table 1). We stratified this cohort in the same way as the HIV cohort, and 'followed up' this cohort, too, for any subsequent record of MS. As with the HIV cohort, anyone with a record of MS prior to or at the same time as the first admission for a reference

Table 1 Age distribution of people entering the HIV cohort, the percentage who were female, and the number of people who entered the reference cohort*

\begin{tabular}{lcclc}
\hline $\begin{array}{l}\text { Age } \\
\text { (years) }\end{array}$ & $\begin{array}{l}\text { HIV cohort } \\
\text { Number } \\
\text { (\% of total) }\end{array}$ & & $\begin{array}{l}\text { Female } \\
\text { (\%) }\end{array}$ & $\begin{array}{l}\text { Reference } \\
\text { cohort } \\
\text { Number }\end{array}$ \\
\hline $0-4$ & 139 & $(0.7)$ & 44.6 & 112355 \\
$5-9$ & 168 & $(0.8)$ & 45.2 & 145618 \\
$10-14$ & 138 & $(0.7)$ & 57.2 & 113372 \\
$15-19$ & 173 & $(0.8)$ & 59.0 & 166198 \\
$20-24$ & 653 & $(3.1)$ & 44.4 & 200079 \\
$25-29$ & 2136 & $(10.1)$ & 43.9 & 264771 \\
$30-34$ & 4066 & $(19.2)$ & 36.9 & 377462 \\
$35-39$ & 4792 & $(22.6)$ & 29.6 & 444962 \\
$40-44$ & 3701 & $(17.5)$ & 24.9 & 390356 \\
$45-49$ & 2228 & $(10.5)$ & 20.6 & 329573 \\
$50-54$ & 1293 & $(6.1)$ & 18.6 & 345117 \\
$55-59$ & 776 & $(3.7)$ & 17.4 & 375702 \\
$60-64$ & 480 & $(2.3)$ & 16.3 & 377503 \\
$65-69$ & 274 & $(1.3)$ & 21.2 & 387109 \\
$70-74$ & 98 & $(0.5)$ & 21.4 & 397229 \\
$75-79$ & 37 & $(0.2)$ & 27.0 & 385998 \\
$80-84$ & 22 & $(0.1)$ & 22.7 & 282933 \\
$85+$ & 33 & $(0.2)$ & 45.5 & 202159 \\
All ages & 21207 & $(100)$ & 30.2 & 5298496 \\
\hline
\end{tabular}

Note that all eligible controls were used (there is no merit in discarding controls to equalise numbers in each age group); and that all analyses were done within strata (eg, the 112355 controls aged 0-4 were compared with the 139 in the HIV cohort). The expected numbers in each stratum were then summed to give age-standardised comparisons of expected and observed numbers with MS in each cohort.

*The reference cohort consisted of people admitted with the following conditions coded under the Office of Population, Censuses and Surveys code (OPCS) edition 4 for operations and the ICD revision 10 for diagnoses: adenoidectomy (OPCS4 E20), tonsillectomy (F34+F36), appendectomy (H01-H03), total hip replacement (W37W39), total knee replacement (W40-W42), cataract (ICD 10 H25), otitis externa/ media (H60-H67), varicose veins (I83), haemorrhoids (I84), deflected septum, nasal polyp (J33+J34.2), inguinal hernia (K40), gallbladder disease (K80-K81), in-growing toenail and other diseases of nail (L60), sebaceous cyst (L72.1), bunion (M20.1) internal derangement of knee (M23).

MS, multiple sclerosis. condition was excluded from the reference cohort. This excluded 877 people with prior MS and 1767 people with a simultaneous record of a reference condition and MS.

Expected numbers of people with MS were determined in each stratum of each cohort, by calculating the rate of MS in the combined population of the HIV and reference cohorts, based on person-days at risk; we then applied that rate to the persondays at risk in the corresponding stratum in, first, the HIV cohort and, second, the reference cohort. This gave stratumspecific expected numbers of people with MS in the HIV and the reference cohort. The expected numbers were added to give all-strata totals in each cohort, which were compared with the observed numbers. Stratum-standardised summary estimates of relative risk were then calculated according to standard statistical practice described elsewhere. ${ }^{5}$ The rate ratio (RR) was calculated using the formula $\left(\mathrm{O}^{\mathrm{HIV}} / \mathrm{E}^{\mathrm{HIV}}\right)$ : $\left(\mathrm{O}^{\mathrm{ref}} / \mathrm{E}^{\mathrm{ref}}\right)$, where the Os and Es are the observed and expected numbers of people with MS in, respectively, the HIV cohort and the reference cohort.

Subanalyses were performed, restricting the outcome of MS to include only the people whose first record of MS, known to us, was more than a year, and then more than 5 years, after the first record of HIV. The reason for this was to identify the sequence of HIV occurring before MS as best we could. In addition, we performed separate analyses for each ethnic group.

\section{RESULTS}

The total number of people who entered the HIV cohort was 21207 (152618 person-years at risk); the total number of people who entered the reference cohort was 5298496 (39 998613 person-years at risk). The median follow-up period for the HIV cohort was 2454 days (IQR 1790 days); the median follow-up period for the reference cohort was 2756 days (IQR 1951 days). Table 1 shows the age distribution of the HIV cohort, the percentage who were women, and the number of people who entered the reference cohort. The distribution of all other variables by which we stratified the cohorts in the analyses are shown in the online supplementary appendix table. Overall, the RR of MS in people with HIV, relative to those without HIV, was 0.38 (95\% CI 0.15 to 0.79 ), based on 7 observed and 18.3 expected cases (table 2). Restricting the outcome to include only the people whose first record of MS, known to us, was more than a year after the first record of HIV, the RR was 0.25 (95\% CI 0.07 to 0.65 ), based on 4 observed and 15.8 expected cases. Restricting the outcome to include only the people whose first record of MS was more than 5 years after the first record of HIV, the RR was $0.15(95 \% \mathrm{CI}<0.01$ to 0.83$)$, based on 1 observed and 6.7 expected cases (table 2).

Table 2 Observed (MS obs) and expected (MS exp) numbers of people in the HIV cohort who had a subsequent record of multiple sclerosis (MS), shown by age at entry to the HIV cohort and by time interval from the first HIV record to the first MS record; rate ratios and $95 \% \mathrm{Cls}$

\begin{tabular}{lllll}
\hline $\begin{array}{l}\text { Age at entry to } \\
\text { HIV cohort (years) }\end{array}$ & $\begin{array}{l}\text { Time interval } \\
\text { (years) }\end{array}$ & MS obs & MS exp & RR $(95 \%$ Cl) \\
\hline All & All & 7 & 18.3 & $0.38(0.15$ to 0.79$)$ \\
All & $1+$ & 4 & 15.8 & $0.25(0.07$ to 0.65$)$ \\
All & $5+$ & 1 & 6.7 & $0.15(<0.01$ to 0.83$)$ \\
$<45$ & All & 3 & 13.9 & $0.22(0.04$ to 0.63$)$ \\
\hline
\end{tabular}


When confining the analysis to people whose ethnicity was recorded as White British, the RR was 0.37 (95\% CI 0.08 to 1.08), based on 3 observed and 8.1 expected cases in the HIV cohort. When we confined the analysis to other ethnic groups, numbers were small. Furthermore, data on ethnicity were often recorded as 'not known' or not recorded at all. Combining all other specified ethnic groups, there were 1 observed and 6.8 expected MS cases in a cohort of 5353 people; where ethnicity was either recorded as 'not stated' or where ethnicity was not recorded at all, there were three observed and four expected cases in an HIV cohort of 10849 people.

\section{Sensitivity analyses}

We repeated the analyses including the cases with MS on the first record of HIV or reference condition. The observed number of cases of MS in the HIV cohort rose to 10, the expected to 22.4 , and the RR was 0.45 (0.21-0.82). We re-ran the analyses excluding people aged 70 and over from both cohorts in case the upper age groups, with fewer people, had any distorting effect. The RR was $0.34(0.12-0.74)$, based on 6 observed and 17.7 expected cases.

\section{DISCUSSION}

Our findings are consistent with the national study in Denmark, but, crucially, given the size of the cohorts in the exposure as well as control groups in our study, the negative association between HIV and MS was statistically significant. If subsequent studies demonstrate there is a causal protective effect of HIV (and/or its treatment), and if the magnitude of it proves to be similar to our rate ratio of 0.38 , this would be the largest protective effect of any factor, yet observed in relation to the development of MS. Previous studies have shown that factors such as smoking, ${ }^{6-8}$ specific gene markers, ${ }^{9}{ }^{10}$ vitamin D deficiency ${ }^{11} 12$ and many different viruses ${ }^{13-16}$ increase the risk of either acquiring MS or of MS progressing, compared with controls. However, not having these risks may not be protective if the relative risk, compared with the general population, simply returned to 1 .

There are several possible explanations for the observed association in our study.

First, immunodeficiency induced by HIV itself (even in the absence of antiretroviral treatment) may prevent development of MS. HIV impairs immune cell homoeostasis and targets a wide range of immune cells and signalling pathways overlapping with MS pathogenesis. Second, antiretroviral medications used to suppress HIV replication potentially may suppress other viral pathogens implicated in MS, for instance HERVs and herpes viruses. ${ }^{17}$

If having HIV is associated with a significantly lower risk of subsequent MS and the association is in the causal chain, it is either because of some biological effect of HIV itself on the pathogenesis of MS, or because the treatment for HIV are coincidentally also treating or preventing development of MS. Unfortunately, neither this study nor any study design that is feasible at present can prove which mechanism may be correct. However, we have made some reasonable assumptions, below, to assist in furthering this issue.

The pendulum of when to start HIV therapy has swung over the past two decades from early treatment, in order to preserve the remaining CD4 cells to later treatment, when it was observed that the CD4 counts recovered when HIV load was suppressed and now back to early treatment in the most recent WHO recommendations. As the exposure (HIV) cohort was determined during or after 1999 , it is probable that the majority of patients would be taking highly active antiretroviral therapy (HAART), which started to become widely used in the UK after 1995. At that time, the trend in clinical management of patients with HIV was to recommend start of cART as early as possible ${ }^{18}$ and therefore it is likely that most of the exposed cohort were taking antiretroviral therapies. This assumption is supported by the global meta-analysis of temporal trends in CD4 cell counts from 1992 to 2011 that determined the mean CD4 cell count among HIV-positive patients in the UK was around 300 cells/ $\mu \mathrm{L}$, which was during our period of observation, and this was a level at which it is most likely cART would have been started. ${ }^{19}$ It is also probable that a number of patients were first identified as having HIV infection during the hospital admission when they entered the HES cohort. In order to further test the hypothesis that cART restricts the development of MS, we investigated the RR of MS cases in the exposed cohort relative to the reference cohort 1 and 5 years, after the initial admission for HIV or reference condition. These data revealed a further reduction in the RR from 0.38 (95\% CI 0.15 to 0.79$)$ overall, to 0.25 (95\% CI 0.07 to $0.65 ; \mathrm{p}<0.005$ ) after 1 year, and 0.15 $(95 \% \mathrm{CI}<0.01$ to $0.83 ; \mathrm{p}=0.04)$ after 5 years. The overlapping CIs of these point estimates mean that these differences are not statistically significant. However, if the progressive decline in risk over time is real, a possible explanation is that during the first admission for HIV some patients were started on cART and the effect of cART on the risk of MS did not begin until an undetermined time period after discharge. Our data, indicating the possible protective effect of cART increases at least 1 year after the HIV indicator admission, may support this observation.

\section{Strengths and weaknesses}

Strengths of our study are that it is national, in a population of about 55 million and that we compared the exposed (HIV) cohort with a large number of controls. The very large number of controls per exposed person in each stratum (approximately $300: 1$ ), and the wide range of different control conditions, would dilute any single factor that may bias the comparison of the HIV and reference cohorts. The very large number of controls also allowed for precise calculation of the number of cases of MS expected in the HIV cohort. Given that the hypothesis is that there would be few cases of MS in the HIV cohort, precision in estimating the number of expected cases is particularly important. Weaknesses of this type of study are numerous. Data on ethnicity were sometimes missing. However, even though we did not have ethnicity data for every participant we demonstrated that the deficit appears not to be restricted to one ethnic group. Our results are consistent with the pattern of ethnicity of the HIV population in the UK, which is predominantly Caucasian men. We have assumed that the time intervals between the first record of HIV and the first record of MS, as known to us, are generally reliable, but we have no way of knowing this is absolutely certain.

We lack data on the proportion of the exposed population who were actually taking cART and the exact combination of drugs they may have been taking during the period of observation. We assume, from what is known about clinical practice during the 12 years of data collection, that the majority of HIV patients were probably either already on cART at the time of entering the exposed cohort or were started on cART if HIV was confirmed at the time of the first admission.

The ideal design for a study of the type reported would be a randomised controlled intervention study where HIV-positive patients are randomised to cART or no cART and followed to 
determine their risk of developing MS. Clearly, this study is not feasible. Another option would be to try and investigate what happened in the pre-cART time from 1981 when HIV was first described, until 1995, when cART was introduced. Unfortunately, this is also not possible as there are no cohorts large enough, in that era, to give a reasonable incidence of MS. Moreover, there is always a possibility of misdiagnosis. HIV infection may clinically present as MS-like symptoms with production of oligoclonal bands in cerebrospinal fluid indistinguishable from MS. In the pre-cART era, with no gadolinium-enhancing MRI available, patients with HIV who presented with MS-like symptoms would generally have been thought to have an HIV-related neurological condition rather than diagnosed as having MS. HIV itself may cause various diseases of the white matter, in addition to an opportunistic infection, such as progressive multifocal leukoencephalopathy. If, in reality, patients had MS, they would most likely have died before the MS would become apparent. When cART became available around 1995, any patients with HIV, presenting with MS-like symptoms, would be assumed to have an HIV neurological condition and immediately started on cART. If MS were the true underlying cause of their condition and their MS symptoms persisted even following cART, one would expect they would be diagnosed with MS, while their HIV was controlled.

The unresolved question is that after almost 20 years of available cART, why do there seem to be almost no documented cases of patients, in the literature so far, with coexisting MS and HIV? Given that both MS and HIV result affect the immune system, it is interesting to note that no pharmaceutical companies who produce therapies for either HIV or MS (no companies produce therapies for both HIV and MS) have guidelines on how to treat patients who have HIV and MS. In fact, medical and marketing personnel at companies that produce MS disease modifying treatments (DMTs) do not recall ever receiving an inquiry about how to use these DMTs in patients with HIV who are taking cART (personal communication).

\section{Conclusion}

This report is the largest record linkage study undertaken to investigate a possible association between HIV and MS. Our investigation revealed that having HIV, and presumptively being on HAART, provided a significant and potentially protective effect in relation to the risk of development of MS. The magnitude of this effect $(>60 \%)$ is at the highest level of any prognostic risk factor investigated to date. Nonetheless, there are inevitable methodological uncertainties in our study design and our findings should be regarded as speculative rather than definitive. We have had to make reasonable assumptions about the likelihood of our exposed HIV cohort being treated with cART during the period of observation. Further consideration may also be warranted on conducting other proof-of-concept studies on using antiretroviral drugs in patients with different types of MS. The first clinical study with Raltegravir in patients with relapsing remitting MS is already recruiting in the UK. ${ }^{20}$ Further investigation of our finding has the potential, after more than 170 years since MS was first described by Jean-Martin Charcot, to help reveal the aetiology of MS.

Contributors All authors fulfil the authorship requirements and have approved the final version of the manuscript. JG, HM and GG developed the research question.
$J G, R G, M G$ and $D Y$ developed the study design in consultation with the other authors. RG, MG and DY conducted the data preparation and data analysis. JG, RG, $M G$ and HM wrote the first draft of the manuscript to which all authors made significant subsequent contributions. GG provided practical guiding in-put to the study as well as to the writing and review process.

Competing interests None.

Ethics approval Ethical approval for the construction and analysis of the linked data set was granted by the Central and South Bristol Research Ethics Committee (ref 04/Q2006/176).

Provenance and peer review Not commissioned; externally peer reviewed.

Data sharing statement Additional data can be generated from the HES database if requested.

Open Access This is an Open Access article distributed in accordance with the Creative Commons Attribution Non Commercial (CC BY-NC 3.0) license, which permits others to distribute, remix, adapt, build upon this work non-commercially, and license their derivative works on different terms, provided the original work is properly cited and the use is non-commercial. See: http://creativecommons.org/ licenses/by-nc/3.0/

\section{REFERENCES}

1 Maruszak H, Brew BJ, Giovannoni G, et al. Could antiretroviral drugs be effective in multiple sclerosis? A case report. Eur J Neurol 2011;18:e110-11.

2 Perron $\mathrm{H}$, Geny $\mathrm{C}$, Laurent $\mathrm{A}$, et al. Leptomeningeal cell line from multiple sclerosis with reverse transcriptase activity and viral particles. Res Virol 1989;140:551-61.

3 Blond JL, Beseme F, Duret L, et al. Molecular characterisation and placental expression of HERV-W, a new human endogenous retrovirus family. J Virol 1999;73:1175-85.

4 Nexo BA, Pedersen L, Sorensen HT, et al. Treatment of HIV and risk of multiple sclerosis. Epidemiology 2013;24:331-2.

5 Breslow NE, Day NE. Statistical methods in cancer research. Volume II-the design and analysis of cohort studies. IARC Sci Pub/ 1987:94-5.

6 Manouchehrinia A, Tench CR, Maxted J, et al. Tobacco smoking and disability progression in multiple sclerosis: United Kingdom cohort study. Brain 2013;136:2298-304.

7 Ramagopalan SV, Lee JD, Yee IM, et al. Association of smoking with risk of multiple sclerosis: a population-based study. J Neurol 2013;260:1778-81.

8 Sundström P, Nyström L, Hallmans G. Smoke exposure increases the risk for multiple sclerosis. Eur J Neurol 2008;15:579-83.

9 Lill CM, Schjeide BM, Graetz C, et al.; International Multiple Sclerosis Genetics Consortium. MANBA, CXCR5, SOX8, RPS6KB1 and ZBTB46 are genetic risk loci for multiple sclerosis. Brain 2013;136:1778-82.

10 Lill CM, Schjeide BM, Graetz C, et al. The genetic basis of multiple sclerosis: a model for MS susceptibility. BMC Neurol 2010;10:101.

11 Munger KL, Levin LI, Hollis BW, et al. Serum 25-hydroxyvitamin D levels and risk of multiple sclerosis. JAMA 2006;296:2832-8.

12 Ebers GC, Sadovnick AD, Veith R. Vitamin D intake and incidence of multiple sclerosis. Neurology 2004;63:939.

13 Ascherio A, Munger KL, Lennette ET, et al. Epstein-Barr virus antibodies and risk of multiple sclerosis: a prospective study. JAMA 2001;286:3083-8.

14 Marrie RA, Wolfson C. Multiple sclerosis and varicella zoster virus infection: a review. Epidemiol Infect 2001;127:315-25.

15 Kang JH, Sheu JJ, Kao S, et al. Increased risk of multiple sclerosis following herpes zoster: a nationwide, population-based study. J Infect Dis 2011;204:188-92.

16 Rosche B, Laurent S, Conradi S, et al. Measles IgG antibody index correlates with T2 lesion load on MRI in patients with early multiple sclerosis. PLOS ONE 2012;7: e28094.

17 Nadal M, Mas BJ, Blanco AG, et al. Structure and inhibition of herpesvirus DNA packaging terminase nuclease domain. Proc Natl Acad Sci USA 2010;107:16078-83.

18 Writing Committee on behalf of the British HIV Association (BHIVA). BHIVA guidelines for the treatment of HIV-infected adults with antiretroviral therapy. HIV Med 2001;1:76-101.

19 Lesko CR, Cole SR, Zinski A, et al. A systematic review and meta-regression of temporal trends in adult $\mathrm{CD}^{+}$cell count at presentation to HIV care, 1992-2011. Clin Infect Dis 2013;57:1027-37.

20 Giovannoni G. Raltegravir (Isentress) Pilot Study in Relapsing Multiple Sclerosis (INSPIRE). ClinicalTrials.gov registration number: NCT0176770. http://clinicaltrials. gov/show/NCT0176770 (accessed 5 Feb 2014). 